\title{
Correspondência entre estado antropométrico, variáveis sociodemográficas e de estilo de vida em universitários
}

\author{
Correspondence between anthropometric status, sociodemographic and lifestyle variables \\ in university students
}

\author{
Jéssica Santos Santana ${ }^{1}$ \\ Orcid: https://orcid.org/0000-0002-9073-4096 \\ Manuela Santos Farias ${ }^{3}$ \\ Orcid: https://orcid.org/0000-0002-9923-950X
}

\author{
Sulamita Oliveira Gonzaga ${ }^{2}$ \\ Orcid: https://orcid.org/0000-9991-6833-4734 \\ Maria da Conceição Pereira da Fonseca ${ }^{4}$ \\ Orcid: https://orcid.org/0000-0003-0325-7162
}

Carlos Rodrigo Nascimento de Lira
Orcid: https://orcid.org/0000-0001-7266-1367

\begin{abstract}
Resumo
Introdução: Alterações no estilo de vida ocorrem entre jovens após ingressarem no ensino superior por diversos motivos. Assim, os universitários manifestam fatores de risco para ganho de peso, tais como inatividade física, alterações do sono, alterações dos hábitos alimentares, início para experimentação de substâncias psicoativas como álcool, tabaco e drogas ilícitas, ou seja, práticas que contribuem para o excesso de peso. Objetivo: Avaliar a correspondência entre estado antropométrico, variáveis sociodemográficas e de estilo de vida em universitários. Métodos: Trata-se de um estudo transversal realizado com 155 universitários, maiores de 18 anos, com aplicação de questionário para obtenção de informações sociodemográficas e de estilo de vida. A avaliação antropométrica consistiu da aferição do peso, estatura, dobras cutâneas, circunferência da cintura e do quadril. As análises consistiram em frequência simples e absoluta, além da Análise de Correspondência Múltipla. Resultados: Houve associação entre excesso de peso com indivíduos de idade 26 a 34 anos, sexo masculino, sem outras ocupações, classe econômica baixa, que não desenvolviam atividade doméstica e serem dos cursos bacharelado interdisciplinar e de pós-graduação. Conclusão: Por meio da correspondência observada entre as variáveis de interesse, sugerimos adoção de práticas para promoção de adequados hábitos alimentares entre os universitários, principalmente a partir de ações de Educação Alimentar e Nutricional.
\end{abstract}

Palavras-chave: sobrepeso; antropometria; serviços de alimentação.

\begin{abstract}
Introduction: Changes in lifestyle occur among young people after entering higher education for several reasons. Thus, university students manifest risk factors for weight gain, such as physical inactivity, changes in sleep, changes in eating habits, beginning to experiment with psychoactive substances such as alcohol, tobacco and illicit drugs, that is, practices that contribute to excess of weight. Objective: to evaluate a correspondence between anthropometric status, sociodemographic and lifestyle variables in university students. Methods: This is a cross-sectional study carried out with 155 university students, over 18 years old, with the application of a questionnaire to obtain sociodemographic and lifestyle information. The anthropometric evaluation consisted of measuring weight, height, skinfolds, waist and hip circumference. The analyzes consisted of simple and absolute frequency, in addition to the Multiple Correspondence Analysis. Results: There was an association between being overweight aged 26 to 34 years old, male, without other occupations, low economic class, who did not develop domestic activity and were from the interdisciplinary and postgraduate bachelor courses. Conclusion: Through the correspondence observed between the variables of interest, we suggest the adoption of practices to promote the adaptation of eating habits among university students, mainly through actions of Food and Nutrition Education.
\end{abstract}

Keywords: overweight; anthropometry; food services.

\footnotetext{
${ }^{1}$ Universidade Federal da Bahia. E-mail: jeusanta@gmail.com

${ }^{2}$ Universidade Federal da Bahia. E-mail: sulamitagonzaga@gmail.com

${ }^{3}$ Universidade Federal da Bahia. E-mail: mannufarias0712@gmail.com

${ }^{4}$ Universidade Federal da Bahia. E-mail: mcfonseca@gmail.com

${ }^{5}$ Universidade Federal da Bahia E-mail: carlos.rodrigo.n@hotmail.com
} 


\section{Introdução}

O excesso de peso é definido como acúmulo excessivo de gordura corporal ${ }^{1}$. Em 2014, mais de 1,9 bilhão de adultos estavam com excesso de peso no mundo, sendo 600 milhões em níveis de obesidade. Em 2019, 55,4\% dos brasileiros apresentavam sobrepeso e 20,3\% obesidade. Em Salvador-Bahia, 51,8\% da população adulta apresentava sobrepeso e $18,1 \%$ obesidade $^{2}$. Para desenvolvimento do excesso de peso múltiplos fatores estão envolvidos, dentre os quais se destacam fatores genéticos, endócrinos, estilo de vida e emocionais. Concernente ao estilo de vida, essas mudanças são expressas na alteração do padrão alimentar, redução da atividade física, prática de tabagismo e consumo excessivo de bebidas alcoólicas, dentre outros ${ }^{3,4}$.

Mudanças no estilo de vida ocorrem entre jovens após ingressarem no ensino superior em razão, por exemplo, da alta exigência de rendimento e dedicação, gestão própria de suas finanças, gerenciamento do seu tempo, etc. Assim, os universitários manifestam fatores de risco para ganho de peso, tais como inatividade física e sedentarismo, alterações do sono, ansiedade, estresse e principalmente alterações dos hábitos alimentares ${ }^{5-7}$. Estudos também apontam para outras mudanças no estilo de vida que ocorrem nesta população, como frequentar festas/baladas, início para experimentação de substâncias psicoativas como álcool, tabaco e drogas ilícitas, ou seja, práticas que também contribuem para o excesso de peso $^{8-10}$.

Inúmeras são as consequências que o excesso de peso apresenta, quer seja para o indivíduo, comunidade ou para a economia. Em uma revisão conduzida por Chu et al. ${ }^{11}$ foi identificado que a obesidade e os distúrbios a ela relacionados exerceram grande pressão sobre a economia, além de desencadear questões letais de saúde. No Irã ${ }^{12}$, adolescentes com excesso de peso apresentaram que o auto-estigma relacionado ao peso mediou os efeitos do índice de massa corporal no sofrimento psicológico (IC95\% $=0,08-0,45$ ), qualidade de vida psicossocial (IC95\%=-1,10,-0,32) e na qualidade de vida física (IC95\%=$2,43,-0,26)$. Em um estudo de coorte no Brasil, Padilha et al. ${ }^{13}$ concluíram que a obesidade parental possui efeito transgeracional no peso, ocasionando sobrepeso na prole enquanto crianças até a fase de vida adulta, a obesidade dos pais também foi um fator de risco para a prole apresentar asma quando adultos. Corrêa et $a l .{ }^{14}$ ao estimarem o custo do governo com os cânceres relacionados ao excesso de peso em adultos, considerando os gastos médicos do Sistema Único de Saúde brasileiro encontraram que o custo federal de 2018 para todos os tipos de câncer combinados foi de US\$ 1,73 bilhão, dos quais quase US\$ 710 milhões foram gastos no tratamento do câncer relacionado ao excesso de peso corporal e US\$ 30 milhões foram atribuíveis ao excesso de peso, portanto uma elevada carga econômica para o país.

A alta prevalência do excesso de peso entre universitários ${ }^{15-17}$ evidencia a necessidade de aprofundar o olhar e conhecimento no que concerne esta condição neste grupo, de modo a sinalizar e redirecionar, quando necessário, os hábitos alimentares inadequados, visando ao longo dos anos, uma melhora na saúde e qualidade de vida destes indivíduos. Portanto, estudar a composição corporal dessa população, as condições sociodemográficas e de estilo de vida são de extrema importância, pois determinados hábitos podem se perpetuar quando se tornarem egressos.

Com a transição do perfil nutricional observado na população, a expansão do ensino superior no Brasil e ausência de políticas públicas específicas direcionadas para os universitários levando em consideração suas particularidades, este 
estudo apresenta relevância por tal abordagem $^{18}$. Diante disto, o objetivo deste artigo foi avaliar a correspondência entre estado antropométrico, variáveis sociodemográficas e de estilo de vida em universitários, de forma a avaliar se esses parâmetros apresentam associação ao excesso de peso.

\section{Materiais e Métodos}

\section{Amostra e tipo de estudo}

Estudo transversal, com abordagem quantitativa, desenvolvido com estudantes que frequentavam o Restaurante Universitário (RU) de uma Universidade do nordeste brasileiro. Para o cálculo da amostra, levou-se em consideração a média do Índice de Massa Corporal (IMC) dos participantes, considerando nível de confiança de $95 \%(\alpha=0,05)$ e admitindo-se um erro amostral de $5 \% \quad(d=0,05)$, estabelecendo um n de 136 estudantes. O estudo foi divulgado no RU por meio de cartazes, em redes sociais e via E-mail pela Pró-Reitoria de Ações Afirmativas e Assistência Estudantil da Universidade. No processo de divulgação, ficou estabelecido um período para coleta de dados, logo, quem compareceu até a determinada data, foi atendido, totalizando uma amostra de 155 estudantes. Estudantes de Instituições de Ensino Superior da rede federal brasileira que se alimentam nos RU são considerados beneficiários do Programa Nacional de Assistência Estudantil, independente de pagarem um valor ínfimo pela refeição ou não pagarem nada ${ }^{19}$. A assiduidade destes comensais ao RU é diferente entre os bolsistas e pagantes, assim, decidimos avaliar os parâmetros antropométricos de modo separado entre estes dois grupos.

Este estudo faz parte de um projeto maior, aprovado em Comitê de Ética e Pesquisa da Escola de Nutrição da Universidade Federal da Bahia (parecer $n^{\circ}$ 228.318/2012). Os participantes depois de informados sobre os objetivos do estudo, e aqueles que concordaram em participar, fizeram a leitura e assinatura do termo de consentimento livre e esclarecido.

\section{Critérios de Inclusão e Exclusão}

Os critérios de inclusão foram: realizar no mínimo três refeições por semana no RU; idade igual ou superior a 18 anos; não ser o primeiro semestre frequentando o RU; ser brasileiro; se estrangeiro, que estivesse morando no mínimo há um ano no Brasil. Não foram inclusos: gestantes, por possuir necessidades nutricionais específicas; estudantes estrangeiros com menos de um ano no país; estudante com comprometimento físico que impossibilitasse realização da antropometria; ter iniciado a frequentar o RU no semestre da coleta dos dados.

\section{Procedimentos}

Para obtenção dos dados utilizamos um questionário elaborado pelos pesquisadores e testado em estudo piloto, onde os próprios participantes preenchiam. As variáveis sociodemográficas foram: vínculo com o RU; sexo; faixa etária dividida a partir da mediana; cor da pele; naturalidade; curso; outra ocupação; classe econômica. Para avaliação da classe econômica utilizamos a metodologia da Associação Brasileira de Empresas de Pesquisa $^{20}$. As variáveis referentes ao estilo de vida foram: etilismo; tabagismo; se já realizou ou realiza dieta; motivo para realização de dieta. Informações referentes às atividades desenvolvidas ao longo do dia foram: realiza atividade de lazer; realiza atividade doméstica; horas de atividade de lazer; horas de atividade doméstica; intensidade da atividade de lazer (sedentária, leve, moderada e pesada $)^{21}$; horas de sono.

$$
\text { Para avaliar o estado }
$$
antropométrico, aferimos: peso e estatura e para o cálculo do IMC foram seguidas as recomendações da Organização Mundial da Saúde $(\mathrm{OMS})^{22}$. Para aferir o peso utilizamos balança digital (marca Plenna, modelo MEA 07400) com capacidade de 
$150 \mathrm{~kg}$. A altura foi aferida com estadiômetro (marca SECA, modelo E0123) fixado a 2,20 metros verticais em relação ao piso, em parede sem rodapé. Aferimos a circunferência da cintura (CC) com fita métrica inelástica (marca grafco, modelo \#17-1340-2, 1,50m) seguindo recomendações da $\mathrm{OMS}^{22}$ e interpretamos seguindo os valores de referência para risco de complicações metabólicas associadas à obesidade conforme recomendações da $\mathrm{OMS}^{22}$, em que homens com $\mathrm{CC} \geq 94 \mathrm{~cm}$ foram considerados com risco elevado e $\geq 102 \mathrm{~cm}$, muito elevado e mulheres $\geq 80 \mathrm{~cm}$, como elevado e $\geq 88$ cm muito elevado.

Avaliamos a circunferência do quadril (CQ) com fita métrica inelástica (marca grafco, modelo \#17-1340-2, 1,50m) e seguindo recomendações da $\mathrm{OMS}^{22}$, assim, calculamos a Relação CinturaQuadril (RCQ) a partir dos valores da CC e do quadril e para a classificação deste, utilizamos os pontos de corte da $\mathrm{OMS}^{22}$ : 0,90 para homens e 0,85 para mulheres. Uma relação de 1,0 ou maior, para qualquer sexo, consideramos "em risco". Os padrões de distribuição de gordura corporal foram classificamos segundo Matsuzawa $^{23}$ em andróide e ginóide a partir dos valores da RCQ.

Para a gordura corporal realizamos o somatório das quatro dobras cutâneas (dobra cutânea tricipital, bicipital, subescapular e suprailíaca) obtidas segundo a técnica de Lohman et $a l^{24} \mathrm{e}$ determinamos o percentual de gordura corporal $(\% \mathrm{GC})$ a partir de diferentes idades e sexos, conforme apresentado na tabela de Durnin e Wolmersley ${ }^{25}$. Para aferição das dobras cutâneas utilizamos um adipômetro clínico da Cescorf com sensibilidade de $1 \mathrm{~mm}$ e uma amplitude de leitura de $63 \mathrm{~mm}$.

Todas as medidas foram realizadas em duplicata pelo mesmo examinador, considerando a média aritmética ao final. Nos casos em que a diferença foi maior que $100 \mathrm{~g}$ para peso, $0,2 \mathrm{~cm}$ para estatura,
$\mathrm{CC}$ e CQ e 0,2 $\mathrm{mm}$ para as dobras cutâneas, as medidas foram repetidas.

Para análise dos dados, realizamos análise descritiva (frequência simples e absoluta) para caracterização da amostra, e a análise das relações conjuntas entre variáveis sociodemográficas e de estilo de vida com as antropométricas avaliamos por meio da Análise de Correspondência Múltipla (ACM). A ACM refere-se a uma análise multivariada para a exploração de dados categóricos, utilizada principalmente para verificar, de forma gráfica, relações entre as categorias das variáveis. Nessa metodologia, as categorias das variáveis estudadas foram representadas visualmente por mapa perceptual, e sua correspondência foi avaliada de acordo com a proximidade destas categorias (quanto mais próximas, maior a probabilidade de estarem associadas). Todas as análises foram realizadas no IBM Statistical Package for Sciences versão $21.0^{\circledR}$.

\section{Resultados}

No presente estudo, $56,1 \%$ dos estudantes eram pagantes e $43,9 \%$ eram bolsistas da assistência estudantil. $\mathrm{O}$ sexo predominante foi o feminino em ambos os grupos $(55,2 \%$ dos pagantes vs. $57,4 \%$ dos bolsistas). Em relação à faixa etária, os estudantes estavam entre 18 a 25 anos para ambos os grupos $(82,8 \%$ vs. $61,8 \%)$ e o valor médio das idades foi de 25,1 (DP = 5,2 ) anos para os bolsistas e 22,3 (DP = $4,4)$ anos para os pagantes. Quanto a cor da pele referida, houve predominância da cor $\begin{array}{llll}\text { parda } & (44,8 \% & \text { vs. } & 44,1 \%) \text {. }\end{array}$ Majoritariamente, a naturalidade dos estudantes foi da Bahia $(88,51 \%$ vs. $86,76 \%$ ). Em ambos os grupos, os universitários tinham outras ocupações além da dedicação ao curso acadêmico $(66,7 \%$ vs. $57,4 \%)$. A classe econômica média foi a prevalente entre universitários pagantes $(56,3 \%)$ e bolsistas $(70,6 \%)$. E $32,2 \%$ dos pagantes e $32,4 \%$ dos bolsistas eram da área de humanas. 
Quanto ao estado antropométrico, tendo como parâmetro o IMC, houve predominância de indivíduos em eutrofia $(66,7 \%$ vs. $60,3 \%)$. Tanto a CC $(81,6 \%$ vs. $72,1 \%)$ quanto a RCQ $(86,2 \%$ vs. $83,8 \%)$ dos avaliados não apresentaram riscos para complicações metabólicas associadas à obesidade. Já com relação ao \%GC, constatamos que 21,8\% dos pagantes e $39,7 \%$ dos bolsistas estavam com risco para doenças associadas à obesidade. Verificamos também que a maior parte dos estudantes relatou não ser portador de nenhuma doença crônica, com percentuais acima de $80 \%$ em ambos os grupos (Tabela $1)$.

Tabela 1: Características antropométricas dos universitários e de seus genitores $(\mathrm{n}=155)$. Salvador, Bahia, Brasil, 2019.

\begin{tabular}{|c|c|c|c|c|c|}
\hline \multirow{2}{*}{ Variáveis } & \multirow{2}{*}{ Opções } & \multicolumn{2}{|c|}{ Pagante $(n=87)$} & \multicolumn{2}{|c|}{ Bolsista $(n=68)$} \\
\hline & & $\mathbf{n}$ & $\%$ & n & $\%$ \\
\hline \multirow{4}{*}{ Índice de Massa Corporal } & Magreza & 8 & 9,2 & 5 & 7,4 \\
\hline & Eutrofia & 58 & 66,7 & 41 & 60,3 \\
\hline & Sobrepeso & 15 & 17,2 & 16 & 23,5 \\
\hline & Obesidade & 6 & 6,8 & 6 & 8,9 \\
\hline \multirow{3}{*}{ Circunferência da Cintura } & Aumentada & 10 & 11,5 & 10 & 14,7 \\
\hline & $\begin{array}{l}\text { Risco de complicações } \\
\text { metabólicas }\end{array}$ & 6 & 6,9 & 9 & 13,2 \\
\hline & Sem risco & 71 & 81,6 & 49 & 72,1 \\
\hline \multirow{2}{*}{$\begin{array}{c}\text { Distribuição de gordura } \\
\text { corporal }\end{array}$} & Ginoide & 81 & 93,1 & 65 & 95,6 \\
\hline & Androide & 6 & 6,9 & 3 & 4,4 \\
\hline \multirow{2}{*}{ Razão Cintura-Quadril } & Com risco & 12 & 13,8 & 11 & 16,2 \\
\hline & Sem risco & 75 & 86,2 & 57 & 83,8 \\
\hline \multirow{4}{*}{$\begin{array}{c}\text { Percentual de Gordura } \\
\text { Corporal }\end{array}$} & Abaixo da média & 15 & 17,2 & 11 & 16,2 \\
\hline & Acima da média & 44 & 50,6 & 28 & 41,2 \\
\hline & Média & 9 & 10,3 & 2 & 2,9 \\
\hline & Risco & 19 & 21,8 & 27 & 39,7 \\
\hline \multirow{4}{*}{ Presença de doença } & Diabetes Mellitus & 1 & 1,1 & 1 & 1,5 \\
\hline & Colesterol alto/Hipertensão & 2 & 2,3 & 1 & 1,5 \\
\hline & Nenhuma doença & 78 & 89,7 & 57 & 83,8 \\
\hline & Outras & 6 & 6,9 & 9 & 13,2 \\
\hline
\end{tabular}

Sobre o estilo de vida, foi encontrado um percentual elevado no consumo de bebida alcóolica $(63,2 \%$ vs. $64,7 \%$ ). Ao que se refere o uso de tabaco, a maior parte dos estudantes referiram não fumar $(92 \%$ vs. $88,2 \%)$. Em ambos os grupos, houve predominância na realização de atividades domésticas (92\% vs. 88,2\%) com tempo de realização entre uma a quatro horas por dia ( $85 \%$ vs. $77,9 \%)$ e de atividades de lazer $(75,9 \%$ vs. $73,5 \%)$, sendo dispendida mais de cinco horas diariamente entre os pagantes $(42,5 \%) \mathrm{e}$ uma a quatro horas por dia entre bolsistas $(39,6 \%)$. Contudo, foi constatado alto percentual no nível de intensidade leve das atividades de lazer $(36,8 \%$ vs. $44,1 \%)$. Referente às horas de sono, os estudantes pagante (7 a 8 horas - 49,3\%) referiram dormir mais horas que os bolsistas (4 a 6 horas - 49,9\%). A maioria dos universitários referiu não realizar dieta $(56,3 \%$ vs. $61,8 \%)$ e entre os que já realizaram, a motivação foi para perda de peso $(13,8 \%$ vs. $16,2 \%)$.

A partir da ACM, duas dimensões foram retidas no modelo, os quais apresentaram autovalores de 2,1 e 1,8, com explicação de $16,16 \%$ da variância na primeira dimensão e $14,52 \%$ na segunda. Para a construção do modelo da ACM, foram utilizadas as medidas de discriminação das variáveis (Tabela 2). Com isso, consideraram-se apenas as variáveis cujas medidas foram próximas ou superiores aos valores da inércia da dimensão. Estes dizem respeito à variância explicada pela dimensão, assim obteve-se 0,162 de inércia para a primeira e 0,145 de inércia para a segunda. 
Tabela 2: Medidas de discriminação e coordenadas dos centroides, nas dimensões (1 e 2). Salvador, Bahia, Brasil, 2019.

\begin{tabular}{|c|c|c|c|c|}
\hline \multirow{3}{*}{ Variáveis } & \multicolumn{2}{|c|}{ Medidas de discriminação } & \multicolumn{2}{|c|}{ Coordenadas dos centroides } \\
\hline & \multicolumn{4}{|c|}{ Dimensões } \\
\hline & 1 & 2 & 1 & 2 \\
\hline Classe econômica & 0,001 & $\mathbf{0 , 3 8 0}$ & - & - \\
\hline Classe alta & - & - & $-0,043$ & $-1,011$ \\
\hline Classe baixa & - & - & 0,016 & 0,376 \\
\hline $\begin{array}{l}\text { Idade } \\
\end{array}$ & 0,180 & 0,448 & - & - \\
\hline 18 a 25 anos & - & - & $-0,096$ & $-0,400$ \\
\hline 26 a 34 anos & - & - & $-0,109$ & 1,159 \\
\hline$>34$ anos & - & - & 1,817 & 0,922 \\
\hline Excesso de peso & 0,026 & 0,294 & - & - \\
\hline Sim & - & - & 0,243 & 0,822 \\
\hline Não & - & - & $-0,106$ & $-0,358$ \\
\hline Atividade doméstica & 0,008 & 0,125 & - & - \\
\hline $\operatorname{Sim}$ & - & - & $-0,029$ & $-0,116$ \\
\hline Não & - & - & 0,267 & 1,080 \\
\hline Atividade de lazer & 0,644 & 0,012 & - & - \\
\hline Não & - & - & 1,384 & $-0,192$ \\
\hline Sim & - & - & $-0,465$ & 0,065 \\
\hline Intensidade da atividade de lazer & 0,645 & 0,109 & - & - \\
\hline Sedentária & - & - & $-0,043$ & $-0,188$ \\
\hline Leve & - & - & $-0,354$ & $-0,664$ \\
\hline Moderada & - & - & $-0,230$ & 0,332 \\
\hline Pesada & - & - & $-0,840$ & 0,404 \\
\hline Nenhuma & - & - & 1,885 & $-0,360$ \\
\hline Tabagista & 0,144 & 0,001 & - & - \\
\hline Sim & - & - & 1,157 & 0,068 \\
\hline Não & - & - & $-0,124$ & $-0,007$ \\
\hline Etilista & 0,003 & 0,001 & - & - \\
\hline Sim & - & - & $-0,038$ & $-0,020$ \\
\hline Não & - & - & 0,067 & 0,035 \\
\hline Outra ocupação & 0,097 & 0,096 & - & - \\
\hline Sim & - & - & $-0,240$ & $-0,240$ \\
\hline Não & - & - & 0,402 & 0,401 \\
\hline Curso & 0,101 & $\mathbf{0 , 1 1 2}$ & - & - \\
\hline Biológicas & - & - & $-0,327$ & $-0,028$ \\
\hline Exatas & - & - & $-0,033$ & 0,223 \\
\hline Humanas & - & - & 0,429 & $-0,403$ \\
\hline Bacharelado interdisciplinar & - & - & $-0,219$ & 0,498 \\
\hline Pós-Graduação & - & - & 0,178 & 0,682 \\
\hline Cor da pele & 0,226 & 0,052 & - & - \\
\hline Branco & - & - & 0,150 & $-0,472$ \\
\hline Negro & - & - & 0,535 & 0,046 \\
\hline Pardo & - & - & $-0,463$ & 0,129 \\
\hline Outros & - & - & $-1,277$ & 0,617 \\
\hline Bolsista/Pagante & 0,014 & 0,231 & - & - \\
\hline Bolsista & - & - & 0,133 & 0,544 \\
\hline Pagante & - & - & $-0,104$ & $-0,425$ \\
\hline Sexo & 0,015 & 0,028 & - & - \\
\hline Masculino & - & - & $-0,139$ & 0,190 \\
\hline Feminino & - & - & 0,109 & $-0,148$ \\
\hline
\end{tabular}


Verificou-se maior composição e influência na primeira dimensão referente à atividade de lazer, intensidade da atividade de lazer, tabagismo e cor da pele. Já na segunda dimensão, constatou-se influência em relação à classe econômica, idade, excesso de peso, atividade doméstica, curso, ser bolsista ou pagante e sexo (Tabela 2).

A quantidade de variáveis nas dimensões do mapa perceptual se deu em decorrência do processamento dos valores encontrados nas coordenadas dos centroides (Tabela 2), que permitiram a construção dos pontos pelo qual os parâmetros estão inseridos, simbolizados e classificados por sinais positivos e negativos no que se referem às coordenadas dos centroides de cada categoria. As correlações entre categorias de variáveis e formação de conglomerados podem ser avaliadas analisando a proximidade entre os pontos (Figura 1), bem como pelos padrões de relações entre essas características.

Figura 1: Visualização das características sociodemográficas e de estilo de vida dos universitários, a partir do gráfico de pontos de categorias entre as dimensões 1 e 2. Salvador, Bahia, Brasil, 2019.

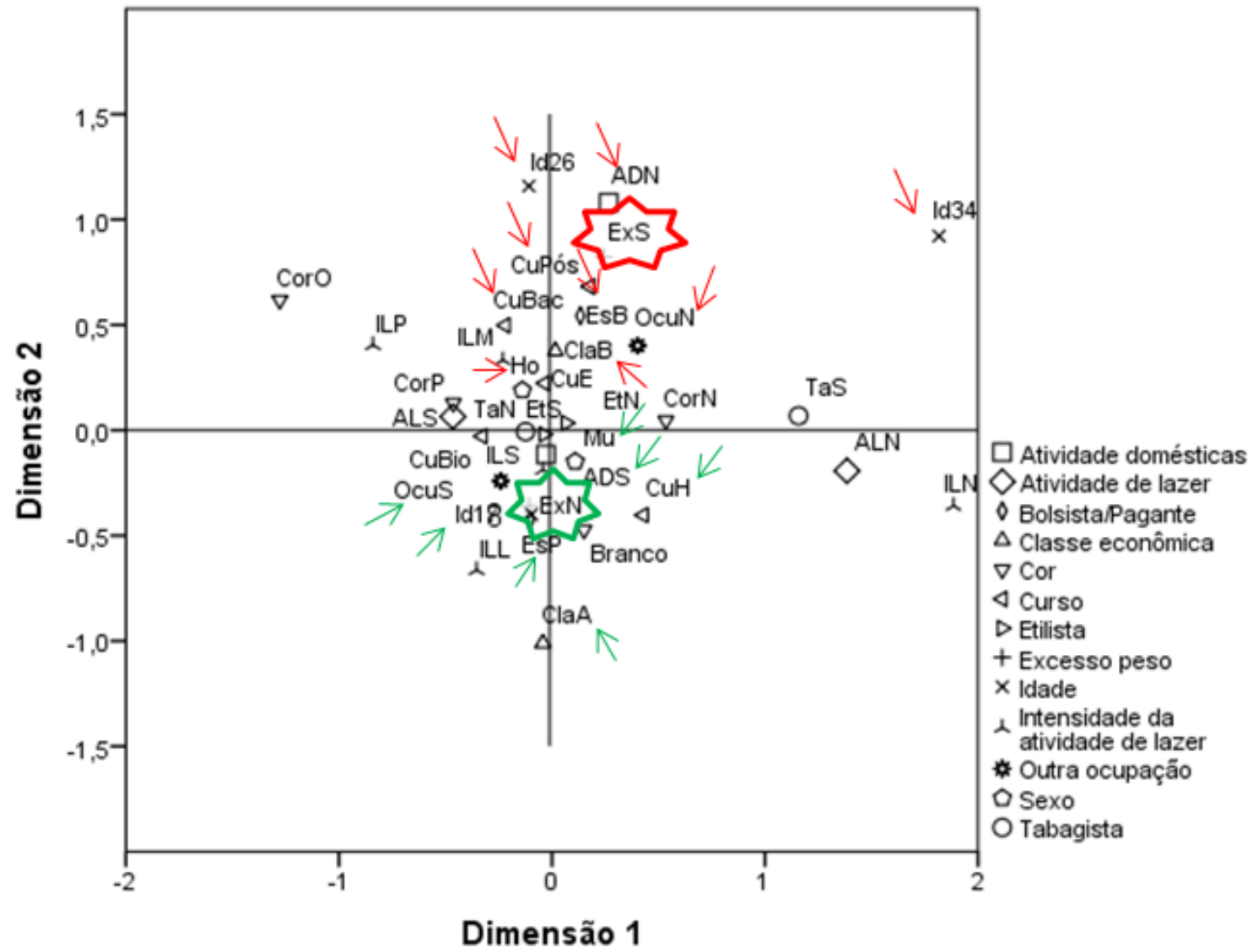

\begin{tabular}{|l|l|l|l|}
\hline \multicolumn{2}{|c|}{ Sigla } & \multicolumn{1}{c|}{ Segenda } \\
\hline \multicolumn{2}{|c|}{} & Exgla & Categoria da variável \\
\hline Faixa etária (idade) & 18 a 25 anos & Excesso de peso & Sim \\
\hline Id 18 & 26 a 34 anos & ExN & Não \\
\hline Id 26 & $>34$ anos & Classe econômica & \\
\hline Id 34 & & ClaA & Classe alta \\
\hline Sexo & Masculino & ClaB & Classe baixa \\
\hline $\mathrm{Ho}$ & Feminino & Etilismo & \\
\hline $\mathrm{Mu}$ & &
\end{tabular}




\begin{tabular}{|c|c|c|c|}
\hline \multicolumn{4}{|c|}{ Legenda } \\
\hline Sigla & Categoria da variável & Sigla & Categoria da variável \\
\hline \multicolumn{2}{|l|}{ Cor da pele } & EtS & Sim \\
\hline $\mathrm{CoB}$ & Branco & EtN & Não \\
\hline $\mathrm{CoN}$ & Negro & Tabagismo & \\
\hline $\mathrm{CoP}$ & Pardo & $\mathrm{TaS}$ & Sim \\
\hline $\mathrm{CoO}$ & Outros & $\mathrm{TaN}$ & Não \\
\hline \multicolumn{2}{|l|}{ Curso } & \multicolumn{2}{|c|}{ Intensidade do lazer } \\
\hline CuBio & Curso biológicas & ILS & Sedentária \\
\hline $\mathrm{CuE}$ & Curso exatas & ILL & Leve \\
\hline $\mathrm{CuH}$ & Curso humanas & ILM & Moderada \\
\hline $\mathrm{CuBac}$ & Curso bacharelado & ILP & Pesada \\
\hline CuPos & Curso de pós-graduação & ILN & Nenhuma \\
\hline \multicolumn{2}{|c|}{ Outra ocupação } & \multicolumn{2}{|c|}{ Atividade doméstica } \\
\hline OcuS & Sim & ADS & Sim \\
\hline OcuN & Não & ADN & Não \\
\hline \multicolumn{2}{|c|}{ Vínculo do estudante } & \multicolumn{2}{|c|}{ Atividade de lazer } \\
\hline EsB & Estudante bolsista & ALS & Sim \\
\hline EsP & Estudante pagante & ALN & Não \\
\hline
\end{tabular}

O excesso de peso está localizado no quadrante superior direito, em ambos os grupos, que influencia o quadrante inferior esquerdo, para estudantes pagantes, e o superior direito, para estudantes bolsistas. Com base no gráfico, estiveram associados ao excesso de peso ser bolsista, ter idade entre 26 a 34 anos, ser do sexo masculino, aqueles que não desenvolviam atividades domésticas, que não possuíam outras ocupações, eram dos cursos de bacharelado interdisciplinar ou de pós-graduação e da classe econômica baixa. Em contrapartida, esteve relacionado ao não excesso de peso as variáveis ser pagante, ser do sexo feminino, ter idade entre 18 a 25 anos, ser do curso de humanas, aqueles que desenvolviam atividades domésticas, ser de classe econômica alta e que tinham outra ocupação (Figura 1).

\section{Discussão}

A partir do método de análise proposto, os resultados da presente investigação indicaram as variáveis distintas entre si e que estiveram relacionadas ou não ao estado antropométrico entre os grupos estudados. Aqui, entendemos o desfecho antropométrico (seja pelo IMC, CC e/ou $\% \mathrm{GC}$ ) como o excesso de peso.
Os fatores determinantes para desenvolvimento do excesso de peso se constituem em um complexo conjunto de fatores genéticos, comportamentais, metabólicos, sociais e psicológicos ${ }^{4}$. Assim, dentre as variáveis que estiveram relacionadas a tal desfecho, observamos que ser bolsista ${ }^{26}$, não ter outra ocupação e pertencer à classe econômica baixa revelase como algo preocupante, pois denota o quanto a vulnerabilidade social é um importante fator que tem impacto direto nos desfechos de saúde. Autores como Pisabarro et $a l^{27}$ e Pires e Mussi ${ }^{18}$, ao discorrerem sobre o nível socioeconômico, acreditam que o mesmo influencia diretamente na prevalência do excesso de peso, sendo predominante nos extratos mais baixos e médios, tendo em vista que o nível socioeconômico pode interferir no acesso a alimentos, à bens e serviços e saúde, resultando assim em menor adoção de hábitos saudáveis.

Este estudo foi desenvolvido com beneficiários do Programa Nacional de Assistência Estudantil ${ }^{19}$ e que utilizavam frequentemente o RU para realização de suas refeições. Os RU têm a função de proporcionar uma refeição nutricionalmente balanceada e em condições higienicossanitárias adequadas. Estes RU, por sua vez, podem minimizar o 
impacto que a saída do ambiente familiar ocasiona, ajudar para as não escolhas alimentares não saudáveis e contribuir para manutenção da saúde dos universitários, quando a alimentação dos estudantes ocorre no âmbito da Universidade, contudo, para o ganho ponderal vale reforçar que a alimentação ao longo do dia deve ser analisada ${ }^{28}$.

Outros fatores como idade entre 26 a 34 anos, ser do sexo masculino, não desenvolver atividade doméstica e serem dos cursos de bacharelado interdisciplinar ou de pós-graduação também estiveram relacionadas ao excesso de peso. A faixa etária e o sexo identificado neste estudo corroboram com pesquisas nacionais ${ }^{2}$ ao apresentarem que o percentual de adultos brasileiros (18-34 anos) com excesso de peso corresponde a $55,4 \%$ da população, $57,1 \%$ de homens e $53,9 \%$ de mulheres. Silva et al. ${ }^{29}$ e Santos et al. ${ }^{30}$ também constataram que os universitários do sexo masculino e com idade igual ou superior a 20 anos apresentaram maiores chances para o excesso de peso. A associação do sexo masculino com o excesso de peso pode se dar através dos comportamentos de saúde inadequados mais associados entre os homens, tais como, tabagismo e alcoolismo, ao passo que mulheres apresentam maior controle com a alimentação, por exemplo ${ }^{30,31}$.

Quanto a área de formação, outros estudos também encontraram associação entre excesso de peso e o curso, como na investigação de Lira Neto et $a .^{32}$ onde $42,3 \%$ dos estudantes do bacharelado em administração apresentaram excesso de peso, bem como no estudo de Mori et al. ${ }^{33}$, ao encontrarem que universitários dos cursos de recursos humanos $(60 \%)$, direito $(49,1 \%)$ e engenharia $(53,6 \%)$ apresentaram maiores prevalências de excesso e peso. Neste estudo, encontramos que $35,2 \%$ dos bolsistas e $35,67 \%$ dos pagantes dos cursos de bacharelado interdisciplinar e da pós-graduação apresentaram excesso de peso (dados não presentados em tabela). $\mathrm{O}$ não desenvolvimento de atividades domésticas também esteve relacionado com excesso de peso. Para a realização de atividades domésticas é necessário esforço físico, consequentemente gerando gasto energético, ainda que pequeno, o que sugere contribuir para não acúmulo de calorias $^{34}$, confirmando assim os resultados de Lins et $a^{35}{ }^{35}$, ao sugeriram que exercer atividade física de lazer e doméstica pareceu ter efeito protetor para o excesso de peso (RP: 0,38 - IC 95\%: 0,17 - 0,83).

Em contrapartida as variáveis relacionadas ao excesso de peso, identificamos que desenvolver atividades domésticas, ser de classe econômica alta e ter outra ocupação foram algumas das variáveis relacionadas ao não excesso de peso, achados reforçados por outras investigações, como as de Lira Neto et al. ${ }^{32}$ e Mori et $a^{33}{ }^{33}$.

Ao que se referem às variáveis antropométricas isoladamente, embora a maioria dos universitários não apresentasse riscos para doenças metabólicas associadas à obesidade, ainda assim uma parcela considerável apresentou excesso de peso (40,7\% sobrepeso e $15,7 \%$ obesidade); risco de complicações metabólicas pela CC $(20,1 \%)$, pelo $\% \mathrm{GC}(61,5 \%)$ e pela RCQ $(30 \%)$; e uma distribuição de gordura corporal do tipo androide (11,3\%). Estes resultados apresentam um cenário preocupante e deve ser monitorado constantemente, pois estudos apontam que o excesso de peso e excesso de adiposidade corporal são de alto risco para o surgimento de doenças crônicas não transmissíveis, mesmo entre adultos jovens ${ }^{36-38}$.

Reforçamos, por tanto, a importância deste estudo, pois apesenta informações que contribuem para a proposição de intervenções em curto, médio e longo prazo. Em curto prazo destacamos a realização de medidas educativas e corretivas na elaboração e/ou preparação dos cardápios, e desta forma oferecer preparações com menor densidade calórica. Em médio prazo, o estudo pode 
auxiliar na construção do contrato firmado entre a Universidade e as concessionárias, dentre aquelas que trabalham com terceirização, para aquisição dos gêneros alimentícios e cardápios ofertados. E em longo prazo, junto com outros estudos, sensibilizar órgãos superiores para que mudanças e diretrizes dietéticas sejam pensadas para esta população e consequentemente reduzir a possibilidade do desenvolvimento de doenças crônicas, assim como, reduzirem gastos com a saúde pública.

\section{Conclusão}

O objetivo deste artigo foi alcançado à medida que identificamos quais variáveis sociodemográficas e de estilo de vida estiveram relacionadas ao excesso de peso ou não entre os universitários. Assim, ressaltamos a importância dos restaurantes universitários, uma vez que são espaços voltados para o fornecimento de refeições nutricionalmente equilibradas e de fácil acesso, por se localizarem dentro dos campi das Universidades. Desse modo, é importante verificar a qualidade da alimentação oferecida nesses estabelecimentos e adotar métodos para promoção de hábitos alimentares adequados dos universitários, por meio de ações e estratégias de Educação e Vigilância Nutricional.

\section{Referências}

1. WHO - World Health Organization. Obesity and overweight. Fact sheet. Updated, June 2016.

2. Brasil. Ministério da Saúde. Secretaria de Vigilância em Saúde. Departamento de Análise em Saúde e Vigilância de Doenças Não Transmissíveis. Vigitel Brasil 2019: Vigilância de fatores de risco e proteção para doenças crônicas por inquérito telefônico: estimativas.

3. WHO - World Health Organization. Diet, nutrition and the prevention of chronic diseases. Geneva: World Health Organization, 2003.

4. ABESO - Associação Brasileira para o Estudo da Obesidade e da Síndrome Metabólica. Diretrizes Brasileiras de Obesidade. 4.ed. São Paulo: Companygraf, 2016.

5. Mota MC, Souza DA, Mello MT, Tufik S, Crispim CA. Estilo de vida e formação médica: impacto sobre o perfil nutricional. Ver. bras. Educ. med., 2012;36(3):358-368. doi: https://doi.org/10.1590/S0100-55022012000500010.

6. Pimentel M, Mata M, Anes E. Tabaco e álcool em estudantes: Mudanças decorrentes do ingresso no ensino superior. Psic., saúde \& doenças., 2013;14(1):185-204.

7. Smolka MLRM, Gomes AP, Batista RS. Autonomia no contexto pedagógico: percepção de estudantes de medicina acerca da aprendizagem baseada em problemas. Rev. bras. educ. med., 2014;38(1):5-14. doi: https://doi.org/10.1590/S0100-55022014000100002.

8. Berto SJP, Carvalhaes MABL, Moura EC. Tabagismo associado a outros fatores comportamentais de risco de doenças e agravos crônicos não transmissíveis. Cad. Saúde Pública., 2010:26(8):1573-1582.

9. Batista ES, Campos TN, Valente FX, Priore SE, Franceschini SCC, Sabarense CM, Peluzio MCG. Impacto do tabagismo e álcool sobre a composição corporal de jovens. Revista Brasileira de Cancerologia., 2011;57(3):355-363. doi: https://doi.org/10.32635/21769745.RBC.2011v57n3.670.

10. Denoth F, Siciliano V, Lozzo P, Fortunato L, Molinaro S. The Association between Overweight and Illegal Drug Consumption in Adolescents: Is There an Underlying Influence of the Sociocultural Environment? Plos One., 2011;6(11):e27358.

11. Chu DT, Nguyet NTM, Dinh TC, Lien NVT, Nguyen KH, Ngoc VTN. et al. An update on physical health and economic consequences of overweight and obesity. Diabetes \& Metabolic 
Syndrome: Clinical Research \& Reviews., 2018;12(6):1095-1100. doi: https://doi.org/10.1016/j.dsx.2018.05.004.

12. Lin CY, Imani V, Broström A, Huus $\mathrm{K}$, Björk $\mathrm{M}$, Hodges EA, Pakpour, AH. Psychological distress and quality of life in Iranian adolescents with overweight/obesity: mediating roles of weight bias internalization and insomnia. Eat Weight Disord., 2020;25:1583-1592. doi: https://doi.org/10.1007/s40519-019-00795-5.

13. Padilha LL, Ribeiro CCC, Nascimento JXPT, Simões VMF, Vitti FP, Cardoso VC. Et al. Lifetime overweight and adult asthma: 1978/1979 Ribeirão Preto Birth Cohort, São Paulo, Brazil. Cad. Saúde Pública., 2020;36(3). doi: https://doi.org/10.1590/0102-311X00041519.

14. Corrêa FSR, Bahia LR, Machado RMQ, Malhão TA, Mendonça EDP, Rosa RDS. et al. Costs of cancer attributable to excess body weight in the Brazilian public health system in 2018. PLoS One., 2021;16:e0247983. https://doi.org/10.1371/journal.pone.0247983.

15. Alahmar U, Murra MS, Menegassi B, Spexoto MCB. Fatores associados ao estresse percebido em universitários. Revista Brasileira de Obesidade, Nutrição e Emagrecimento., 2020;14(85):330-339. doi: http://www.rbone.com.br/index.php/rbone/article/view/1270.

16. Melo P, Vieira R. Percepção e satisfação da imagem corporal em estudantes de um centro universitário de Recife/Pernambuco. Revista De Ciências Da Saúde Nova Esperança, 2020;18(3):196-204. doi: https://doi.org/10.17695/rcsnevol18n3p196-204.

17. Gomes ABS, Fernandes RCS, Machado AD, Abreu ES, Spinelli MGN. Associação entre o hábito de consumo de café da manhã e estado nutricional de estudantes universitários. Univap, 2020;26(50).

18. Pires CGS, Mussi FC. Excesso de peso em universitários ingressantes e concluintes de um curso de enfermagem. Esc. Anna Nery., 2016;20(4):e20160098. doi: https://doi.org/10.5935/1414-8145.20160098.

19. Brasil. Decreto ${ }^{0}$ 7.234, de 19 de Julho de 2010. Dispõe sobre o Programa Nacional de Assistência Estudantil - PNAES. Diário Oficial da União. Brasília, DF: BRASIL. 2010.

20. ABEP - Associação Brasileira de Empresas de Pesquisa. Critério de classificação econômica Brasil. 2018. Disponível em: www.abep.org.

21. FAO, WHO, UNU, Expert Consultation. Report on human energy requirements. Interim Report. Comité de expertos de energía de FAO/OMS/UNU; 2004.

22. WHO - World Health Organization. Obesity: preventing and managing the global epidemic. Report of a World Health Organization Consultation. Geneva: World Health Organization, 2000.

23. Matsuzawa Y. Pathophysiology and molecular mechanisms of visceral fat syndrome: the Japanese experience. Diabetes/Metabolism Research and Reviews., 1998;13(1):3-13. doi: https://doi.org/10.1002/(SICI)1099-0895(199703)13:1<3::AID-DMR178>3.0.CO;2-N.

24. Lohman TG, Roche AF, Martorell R. Anthropometric Standardisation Reference Manual. Champaign, IL: Human Kinetics Books; 1988.

25. Durnin, J.V.; Wolmersley, S. Body fat assessed from total body density and its estimation from skinfold thickness: measurements on 481 men and women age from 16 to 72 years. British Journal of Nutrition., 1974;32(1):77-97.

26. Santos CT. Ações afirmativas no ensino superior: análise do perfil socioeconômico e da experiência universitária de bolsistas do ProUni na PUC-Rio. Rev. Bras. Estud. Pedagog., 2012;93(235). 
27. Pisabarro R, Gutiérrez M, Bermúdez C, Prendez D, Recalde A, Chaftare Y, Manfredi A. Segunda Encuesta Nacional de Sobrepeso y Obesidad (ENSO 2) adultos (18-65 años o más). Rev. méd. urug., 2009;25:14-26.

28. Lima KA, Schwarz K, Menegassi B, Machado TWM, Saldan PC. Escolha alimentar de universitários de uma universidade do Paraná. Visão Acadêmica., 2019;20(1):103-112. doi: http://dx.doi.org/10.5380/acd.v20i1.64682.

29. Silva DAS, Quadros TM, Gordia AP, Petroski EL. Associação do sobrepeso com variáveis sócio-demográficas e estilo de vida em universitários. Ciênc. saúde coletiva., 2011;16(11):4473-4479. doi: https://doi.org/10.1590/S1413-81232011001200020.

30. Santos MC, Angarten VG, Munaro HLR, Pelegrini A. Associação do excesso de peso com variáveis demográficas e atividade física no lazer em universitários. R. bras. Ci. e Mov., 2014;22(3):14-19. doi: http://dx.doi.org/10.18511/rbcm.v22i3.4915.

31. Streb AR, Del Duca GF, Silva RP, Benedet J, Malta DC. Simultaneidade de comportamentos de risco para a obesidade em adultos das capitais do Brasil. Ciênc. saúde coletiva., 2020;25(8):2999-3007. doi: https://doi.org/10.1590/1413-81232020258.27752018.

32. Lira Neto JCG, Silva AP, Costa EPN, Lacerda MGC, Silva ARV, Freitas RWJF. Análise do sobrepeso e da obesidade em estudantes universitários. J Nurs UFPE on line., 2012;6(11):2770-6. doi: 10.5205/reuol.2185-16342-1-LE.0611201220.

33. Mori CO, Cruz KCM, Baptista MS, Lauria VT, Teixeira CVLS. Excesso de peso em universitários: Estudo comparativo entre acadêmicos de diferentes cursos e sexos. RBONE., 2017;11(68):717-721.

34. Caspersen CJ, Powell KE, Christenson GM. Physical activity, exercise and physical fitness. Public Health Reports., 1985;100:126-31.

35. Lins APM, Sichieri R, Coutinho WF, Ramos EG, Peixoto MVM, Fonseca VM. Alimentação saudável, escolaridade e excesso de peso entre mulheres de baixa renda. Ciênc. saúde coletiva., 2013;18(2):357-366. doi: https://doi.org/10.1590/S1413-81232013000200007.

36. Shirasawa $T$, Ochiai H, Yoshimoto T, Nagahama S, Kobayashi M, Ohtsu I. et al. Associations between normal weight central obesity and cardiovascular disease risk factors in Japanese middle-aged adults: a cross-sectional study. Journal of Health, Population and Nutrition., 2019;38(46). doi: https://doi.org/10.1186/s41043-019-0201-5.

37. Someya Y, Tamura Y, Kohmura Y, Aoki K, Kawai S, Daida H. Slightly increased BMI at young age is a risk factor for future hypertension in Japanese men. PLoS One., 2018;13(1):e0191170. doi: https://doi.org/10.1371/journal.pone.0191170.

38. Navarro-Prado S, RioValle JS, Alonso MAM, Aparicio ÁF, Jiménez EG. Stricter Adherence to Dietary Approaches to Stop Hypertension (DASH) and Its Association with Lower Blood Pressure, Visceral Fat, and Waist Circumference in University Students. Nutrients., 2020;12(740). doi:10.3390/nu12030740.

\section{Como citar este artigo:}

Santana JS, Gonzaga SO, Farias MS, Fonseca MCP, Lira CRN. Correspondência entre estado antropométrico, variáveis sociodemográficas e de estilo de vida em universitários. Rev. Aten. Saúde. 2021; 19(69): 27-38. 